\title{
Startup Zahnarztpraxis und chirurgisches Basiswissen
}

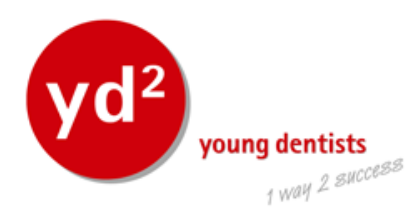

\begin{abstract}
Kurzweilige und fundierte Vorträge aus den Bereichen Betriebswirtschaft und zahnärztlichem Fachwissen standen im April wieder beim "young dentists meeting“ in Bonn auf dem Programm. Es entspricht dem Grundgedanken der Gründer der Initiative (Akademie Praxis und Wissenschaft in der DGZMK und des Freien Verbandes Deutscher Zahnärzte), immer ein interessantes Angebot aus Praxisführung und Fortbildung zu kombinieren. Die Tagungen finden zweimal im Jahr statt.
\end{abstract}

Gerade für den jungen Zahnarzt ist es schwer, eine konkrete Vorstellung seiner beruflichen Zukunft innerhalb einer kurzen Zeit zu entwickeln. Zu vielfältig sind die Optionen: „Einzelkämpfer versus Gemeinschaft“" oder „Neugründung versus Übernahme“ - und das sind nur zwei Beispiele. Hinzu kommen die im Studium und in der Assistenzzeit nur am Rande behandelten Themen wie betriebswirtschaftliche Grundlagen (Gewinn/Rentabilität/Liquidität) und Praxismanagement, die für eine erfolgreiche Praxis unabdingbar sind.

Beim jüngsten „young dentists meeting" hat Diana Brendel, Geschäftsführende Gesellschafterin bei FIBU-doc Praxismanagement GmbH, in ihrem Vortrag „Startup ZahnarztpraxisDer Weg in die Selbstständigkeit" anhand vieler Beispiele gezeigt, wie Berufseinsteiger anhand von wichtigen Faktoren eine Entscheidungsfindung begründen und beschleunigen können, auf welche wichtigen Kennzahlen es ankommt und welche Weichen frühzeitig gestellt werden müssen, um langfristig erfolgreich die eigene Praxis zu führen.

Das gemeinsame Abendessen in der Kamin-Lounge zusammen mit den Referenten bot den Teilnehmern dann die Möglichkeit, in entspannter Atmosphäre noch offene Fragen zu klären. Mit dabei: ein „Ehren-young-dentist“. Der FVDZ-Bundesvorsitzende Harald Schrader war extra aus dem hohen Norden ins Rheinland gereist.

\section{Theoretische Erkenntnisse praktisch ausprobiert}

Der Samstag stand ganz im Zeichen der Chirurgie. Die Tröltzsch Brothers, Dr. Dr. Markus \& Dr. Dr. Matthias Tröltzsch, machten in ihrer unverwechselbaren Art die young dentists fit für die ersten Schritte in der oralen Chirurgie: „Besser mit: Medizinisches und chirurgisches Basiswissen im Praxisalltag- mit praktischen Übungen", lautete ihr Thema.

Im theoretischen Teil vermittelten sie die wichtigsten medizinischen Hintergründe der gesundheitlichen Situation der $\mathrm{Pa}$ tienten, deren multiplen Medikamenteneinnahmen und die daraus immer komplexer werdende orale Chirurgie und damit verbundene Risiken.

Da die Firma Straumann das chirurgische Grundbesteck zur Verfügung stellte, konnten die Teilnehmer die neu erworbenen Kenntnisse von Sites, Schnittführung und Material im praktischen Teil direkt ausprobieren.

Das nächste „young dentists meeting" ist im Oktober, dann steht das Thema Kinderzahnheilkunde im Fokus. Nähere Infos unter www.young-dentists.de.

Christian Honert

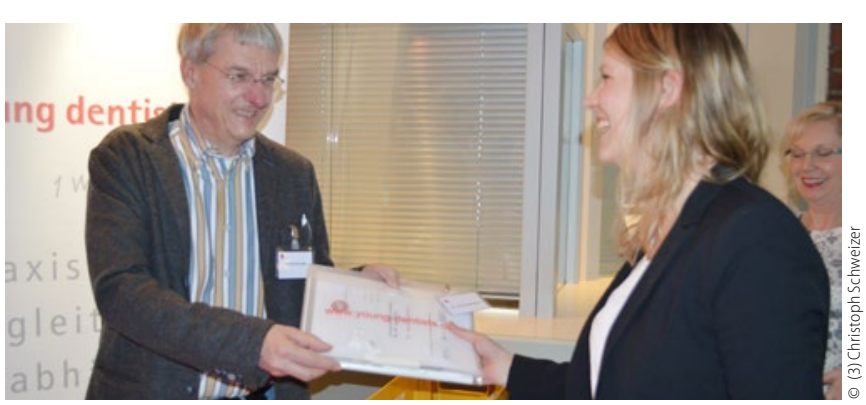

FVDZ-Bundesvorsitzender Harald Schrader zu Gast in Bonn.

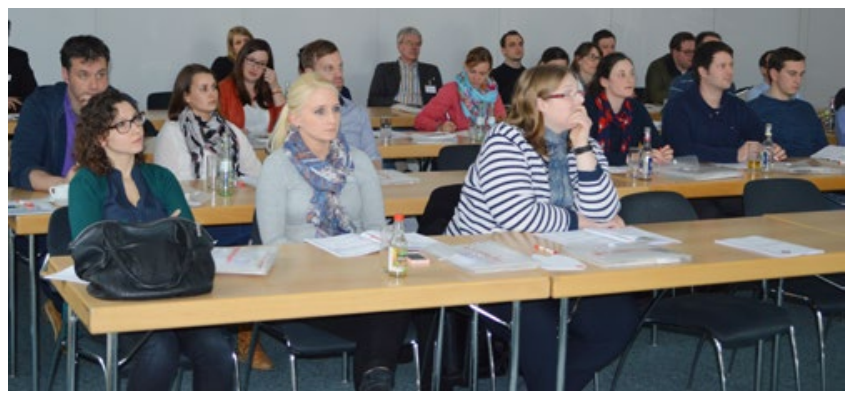

Kurzweilige und fundierte Vorträge interessieren die young dentists.

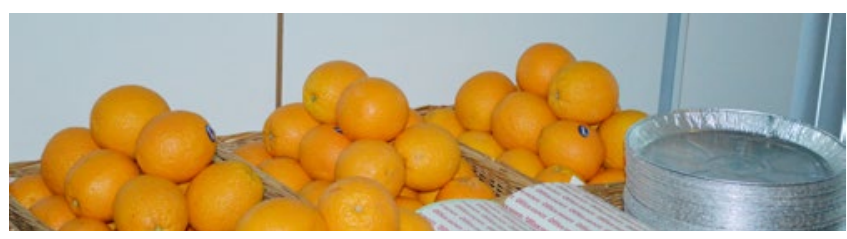

Apfelsinen statt Schweinekiefer für die praktische Übung.

\section{Reaktionen der Teilnehmer}

\section{"Sehr gut!"}

Wie immer wurden die Teilnehmer des „young dentists meeting“ in einer Umfrage nach ihrer Meinung gefragt, wie ihnen die Fortbildung gefallen hat. Und die Reaktionen sind durchweg positiv. Die Antworten reichen von "Sehr gut!" über "Super Referate!" bis hin zum Lob über den Veranstaltungsort. So schrieb ein Teilnehmer: „Das war bisher der beste Raum. Größe und Sichtbarkeit der Präsentation waren sehr gut." Ein anderer kommentierte das Treffen so: „Super Preis-Leistungsverhältnis, angenehme lockere und sehr informative Veranstaltung. War super, vor allem auch die Abwechslung durch die gute praktische Nahtübung." 